\title{
CALIDAD DE VIDA Y DIMORFISMO SEXUAL EN LA POBLACIÓN PREHISPÁNICA DE SAN PEDRO DE ATACAMA (NORTE DE CHILE) ${ }^{1}$
}

\author{
José Cocilovo, Héctor Varela y María Fuchs²
}

\section{* Introducción}

Resumen

La diferencia morfológica entre sexos puede variar reflejando la calidad de vida dominante en una población. La información para San Pedro de Atacama es analizada para evaluar a partir de una muestra de 624 individuos y 35 variables craneométricas. Las diferencias entre sexos se analizaron con las pruebas ANCOVA y MANCOVA para una y dos vías con covariantes, según el caso. El análisis discriminante (DA) permitió evaluar la clasificación del sexo en cada individuo. La mayoría de las mediciones dieron valores medios mayores en los hombres con relación a las mujeres. No obtuvimos pruebas suficientes respecto de la variación del $\mathrm{SD}$ en el tiempo. Esto indica que los cambios en las condiciones de vida registrados durante 60 generaciones con otros marcadores no influyeron sustancialmente en el desarrollo normal del cráneo en ambos sexos.

Palabras claves: dimorfismo sexual - perfil biológico - craneometría San Pedro de Atacama.

Abstract

Morphological differences between both sex, could vary reflecting the dominant life quality in a population. The information available for San Pedro de Atacama is analysed in order to assess the expression of the sexual dimorphism. A sample of 624 individuals and 35 craniometrical variables was utilised. Gender differences were analysed by ANCOVA and MANCOVA tests for one and two-way with covariates, when they apply. Discriminant analysis (DA) allowed the evaluation of the sex assignment of each individual. In most of the measurements higher values in men compared to women were observed. Even the statistical analysis proved the differences between sexes and periods, we couldn't obtain sufficient evidence regarding its variation over time. This indicate that for 60 generations variations in conditions of quality of life, recorded using other markers, substantially not influenced the and normal development of the skull in both sexes.

Key words: sexual dimorphism - biological profile - craniometry San Pedro de Atacama.
El crecimiento y desarrollo normal depende tanto de factores genéticos como ambientales, siendo el dimorfismo sexual (SD) un resultado esperado y una propiedad que distingue a hombres y mujeres en aspectos anatómicos, funcionales y conductuales. La magnitud del mismo, medida como la diferencia entre valores medios masculinos con respecto a los valores medios femeninos (SDI) puede variar por distintas causas relacionadas principalmente con pautas sociales y culturales vinculadas con la obtención, disponibilidad y distribución de los recursos dentro del grupo y entre sexos. Son importantes también, los cambios en el modo de vida, en la economía o en el rol social y laboral de ambos sexos. Entre los factores ambientales, el estrés alimentario crónico, principalmente en las etapas más vulnerables durante la niñez y la adolescencia, influye mayormente en el sexo masculino determinando un menor crecimiento (Pérez et al. 2010) y la reducción de las diferencias con respecto al sexo femenino (Frayer y Wolpoff 1985). En efecto, el déficit en el aporte nutricional (escaso contenido proteico y exceso de carbohidratos) y las malas condiciones sanitarias pueden determinar una reducción dramática del SD (Stini 1969; Kelley y Angel 1987; Dettwyler 1992).

En el hombre, en condiciones normales, el sustento de una mayor masa muscular implica un desarrollo esquelético equivalente, pero en condiciones ambientales desfavorables se produce el retraso del crecimiento masculino comparado con el femenino, circunstancia que permite

Recibido: febrero 2013. Aceptado: mayo 2013.

\footnotetext{
Proyectos: Consejo Nacional de Investigaciones Científicas y Técnicas (CONICET, PIP 2405/08); Secretaría de Ciencia y Técnica-Universidad Nacional de Río Cuarto (SeCyT-UNRC, C18/328); Ministerio de Ciencia y Técnica de la Provincia de Córdoba (Res: 1276/o7); Agencia Nacional de Promoción Científica y Técnica (ANPCYT-FONCYT PICT 02210/07).
} 
evaluar este rasgo en poblaciones modernas y antiguas a partir del análisis de restos esqueletales (Purkait y Chandra 2002, 2004; Kranioti et al. 2008). Particularmente, se dispone de abundante información sobre el cráneo para una amplia región (Perú, Chile y Argentina) y los estudios realizados coinciden en destacar la existencia de diferencias estadísticamente significativas entre hombres y mujeres (Cocilovo 1975, 1978; Cocilovo et al. 1982; Cocilovo y Baffi 1985; Varela et al. 1990; Cocilovo et al. 1994; Cocilovo et al. 2005; Cocilovo et al. 2013; Cocilovo y Varela $2014 \mathrm{Ms}$ ). Sin embargo una reducción marcada del SD fue comprobada en Arica durante los períodos Medio y Tardío (Cocilovo y Varela 2014 Ms) y su vinculación con situaciones de estrés y calidad de vida en Las Pirguas (Salta) (Baffi y Cocilovo 1989; Baffi et al. 1996).

La calidad de vida en la población prehistórica de San Pedro de Atacama fue evaluada en una serie de estudios mediante el análisis de patologías, traumas, estado de la dentición, violencia personal y otros aspectos biantropológicos (Costa 1988; Costa y Llagostera 1994; Costa et al. 1999; Costa et al. 2004; Torres-Rouff et al. 2005; TorresRouff y Costa 2006; Lessa y Mendonça 2007; Da Gloria et al. 2011; Torres-Rouff 2011; Hubbe et al. 2012; Nado et al. 2012). A continuación se expone una síntesis de los trabajos citados.

En el sitio Quitor 6 (940-1240 DC), asignado a una fase tardía del período Medio, se comprobó un "empobrecimiento" cultural, individuos con deformación tabular oblicua y erecta, alta incidencia de caries, enfermedades períodontales y lesiones periapicales, gran abrasión dentaria, alta frecuencia de líneas de Harris e indicadores de violencia, una dieta rica en carbohidratos y esperanza de vida de 26.6 años (Costa 1988). En momentos previos, en Coyo 3 (910-960 DC), los estudios revelaron una mayor frecuencia de deformación tabular erecta, hipoplasia del esmalte, periostitis y osteomielitis, pérdida de piezas dentales, caries y desgaste dental, lesiones periapicales, enfermedades degenerativas, y traumas óseos más frecuentes en mujeres que en varones (Costa y Llagostera

\footnotetext{
2 Departamento de Ciencias Naturales, Facultad de Ciencias Exactas, Físico-Química y Naturales, Universidad Nacional de Río Cuarto, X5804BYA Río Cuarto, y CONICET, ARGENTINA. Email: jcocilovo@exa.unrc.edu.ar; hvarela@exa.unrc.edu.ar; mfuchs@exa.unrc.edu.ar.
}

1994). La integración de la información obtenida para un segmento cronológico más amplio (Solcor 3 pre-Tiwanaku y Tiwanaku, Quitor 6 y Coyo 3 post-Tiwanaku) permitió comprobar que durante la influencia de Tiwanaku, se produjo un aumento de la talla y la duplicación del dimorfismo sexual, indicio de un mejoramiento de la calidad de vida; aunque se observó una menor proporción de caries, el desgaste dental y la hipoplasia del esmalte se mantuvieron constantes, se incrementó la hiperostosis porótica y las señales de traumas y estrés revelaron un alto índice de violencia, sin diferencias entre sexos ni entre fases (Costa et al. 1999, 2004).

Estudios posteriores mostraron un panorama diferente a partir de los recuentos de casos de periostitis y osteomielitis en la misma secuencia de sitios antes mencionados. Las frecuencias fueron semejantes en ambos sexos, alcanzaron mayores valores en los momentos preTiwanaku, Intermedio Temprano o Formativo (Solcor 3) y Tiwanaku o Medio (Solcor 3 Medio), disminuyeron al final de la interacción con esta entidad (Coyo 3) y volvieron a incrementarse en el período Intermedio Tardío o post-Tiwanaku (Quitor 6) (Da Gloria et al. 2011).

Contrastando con la prosperidad establecida para el período Medio (Solcor 3: 750-1000 DC) y durante la dominación incaica (Catarpe: 1476-1532 DC), en el Intermedio Tardío (Coyo 3, Yaye y Quitor 6: 1000-1476 DC), un momento de extrema inestabilidad ambiental y escasez de recursos, la calidad de vida disminuyó sustancialmente, según fue constatado por el incremento de traumas asociados con la violencia interpersonal (Torres-Rouff et al. 2005). Estas observaciones fueron ratificadas contando con una muestra mayor que incluyó materiales de período Intermedio Temprano (Toconao Oriente: 200 AC-600 DC), del Medio, del Intermedio Tardío y Tardío antes citados (Torres-Rouff y Costa 2006). Otros estudios sobre violencia constataron una frecuencia menor en Solcor 3 (pre-Tiwanaku) pero un incremento mayor en Solcor 3 (Tiwanaku) junto con Coyo 3 y Quitor 6 (Lessa 1999; Lessa y Mendonça 2007), lo cual revela situaciones y condiciones de vida diferentes en el período Medio.

Sin embargo, la prosperidad postulada para el período Medio no fue experimentada por todos los individuos, sino solo por algunos, revelando la estructuración de 


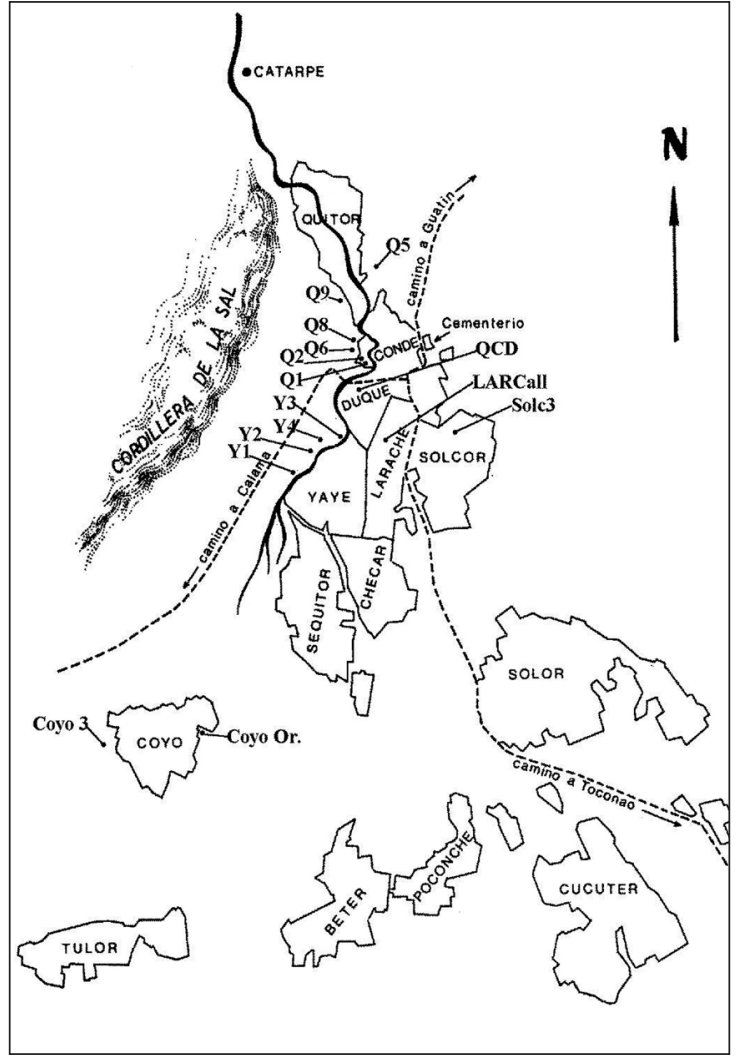

Figura 1. Ubicación de los sitios que integran la muestra: Q1, Q2, Q5, Q6, Q8 y Q9 (Quitor 1, 2, 5, 6, 8 y 9); QCD: Quitor Conde Duque, LARCall: Larrache Callejón, Solcz: Solcor 3 , Coyo 3 y Coyo Oriental. Croquis basado en Le Paige (1963) y en Llagostera y Costa (1999).

la sociedad basada en la desigual disponibilidad de bienes y de recursos alimenticios, inferencia realizada a partir de la prevalencia de indicadores de violencia, caries, desgaste dental y de infecciones inespecíficas (Da Gloria et al. 2011; Torres-Rouff 2011; Hubbe et al. 2012). En el ayllu de Solcor (sitios Solcor 3 y Solcor Plaza), se comprobó la distribución diferencial de ofrendas funerarias, de la deformación artificial, del consumo de alimentos $\left(\delta^{13} \mathrm{C}\right)$ y la presencia de individuos foráneos, con marcas isotópicas $\left({ }^{87} \mathrm{Sr} /{ }^{80} \mathrm{Sr} ; \delta^{18} \mathrm{O}\right)$ superiores a las admitidas para la zona, indicando su origen en el altiplano (Nado et al. 2012).

La variación en los indicadores revelada por las investigaciones citadas tiene que haber dependido de las fluctuaciones del tamaño de la población, situación que fuera prevista a partir del estudio del proceso evolutivo realizado en esta localidad en base a rasgos craneométricos. En efecto, durante el período Medio se postuló un incremento demográfico y la existencia de un mayor flujo génico de mediano y amplio rango (Varela y Cocilovo 1996, 2000, 2009, 2011), lo cual puede haber determinado posteriormente la disminución de la calidad de vida durante los momentos finales del período Medio y en el período Tardío, al superar la capacidad de porte del ecosistema. Un indicador de este proceso es la distribución de sitios registrados en los oasis, cuya proporción se reduce notablemente en el período Tardío comparativamente con respecto a los períodos Medio e Intermedio Tardío (Llagostera y Costa 1999; Agüero 2005). Además, se citan como factores influyentes el empeoramiento del clima por una fuerte sequía y la escasez de alimentos para explicar el incremento de la tasa de infección ósea ( $\mathrm{Da}$ Gloria et al. 2011) asociado con un evento ambiental generalizado establecido a partir de estudios sedimentológicos en la zona del lago Titicaca (Binford et al. 1997).

De acuerdo con los antecedentes expuestos, es razonable pensar que las fluctuaciones de la calidad de vida detectada en la historia de la población pueden haber influido en el crecimiento y desarrollo normal de los individuos, y por lo tanto, en la expresión del SD. El objetivo del presente trabajo es evaluar este rasgo en el cráneo en forma global para la población prehispánica de San Pedro de Atacama y en forma particular tomando en cuenta su distribución en cada período cultural. Se espera que sus variaciones reflejen cambios en las condiciones de vida durante los últimos dos milenios de su evolución biocultural.

\section{* Material y métodos}

La muestra está constituida por 624 individuos adultos, con y sin deformación artificial, depositados en el Instituto de Investigaciones Arqueológicas y Museo R.P. Gustavo Le Paige (San Pedro de Atacama, Chile). El material corresponde a diferentes sitios (Catarpe, Coyo 3, Coyo Oriental, Larache, Quitor 1, Quitor 2, Quitor 5, Quitor 6, Quitor 8, Quitor 9, Quitor CD, Solcor 3, Toconao y Yaye) que cubren los momentos principales del desarrollo agrícola-ganadero de la localidad (Figura 1). Se determinó el sexo (319 masculinos y 305 femeninos) y la edad basados en caracteres morfológicos de acuerdo con Genovés 
(1962) y Buikstra y Ubelaker (1994). La deformación artificial fue clasificada según Dembo e Imbelloni (1938) en tabular (erecta y oblicua), circular (erecta y oblicua) y no deformados. La muestra empleada es similar a la descrita en el trabajo de Varela y Cocilovo (2011) en el cual se exponen los criterios que permitieron la configuración de tres grupos asociados a los componentes Temprano, Medio y

\begin{tabular}{|c|l|c|c|}
\hline Período & \multicolumn{1}{|c|}{ Sitio } & $\mathbf{N}^{\circ}$ & Total \\
\hline \multirow{5}{*}{ Temprano } & Toconao & 17 & \\
& Larache & 15 & 44 \\
& Quitor 8 & 12 & \\
\hline \multirow{5}{*}{ Medio } & Quitor 2 & 56 & \\
& Quitor 5 & 91 & \\
& Quitor 6 & 112 & \multirow{4}{*}{476} \\
& Coyo 3 & 10 & \\
& Coyo Oriental & 161 & \\
& Quitor CD & 26 & \\
& Solcor 3 & 20 & \\
\hline \multirow{5}{*}{ Tardío } & Quitor 1 & 31 & \\
& Yaye & 50 & 104 \\
& Catarpe & 10 & \\
& Quitor CD & 5 & \\
\hline Total & Quitor 9 & 8 & \\
\hline
\end{tabular}

Tabla 1. Dimorfismo sexual: Distribución de la muestra por período y por sitio.

\begin{tabular}{|l|c|c|c|}
\hline \multicolumn{5}{|c|}{ Sexo } \\
\hline \multicolumn{1}{|c|}{ Período } & Masculino & Femenino & Total \\
\hline Temprano & $26(59 \%)$ & $18(41 \%)$ & $44(100 \%)$ \\
\hline Medio & $235(49 \%)$ & $241(51 \%)$ & $476(100 \%)$ \\
\hline Tardío & $58(56 \%)$ & $46(44 \%)$ & $104(100 \%)$ \\
\hline Total & 319 & 305 & $\mathbf{6 2 4}$ \\
\hline
\end{tabular}

Tabla 2. Dimorfismo sexual: Distribución de la muestra por sexo y período. Nota: $x^{2}=2.602$, con 2 gl. $y$ $\mathrm{p}=0.27226$.
Tardío del desarrollo cultural de la localidad (Berenguer et al. 1986; Bravo y Llagostera 1986; Costa 1988; Llagostera et al. 1988; Tarragó 1989; Oakland 1992, 1994; Agüero 2005; Costa et al. 2008).

Se registró la información correspondiente a 35 variables métricas y el análisis de los datos incluyó dos diseños experimentales que cumplen con los objetivos propuestos para el estudio del SD: 1) Muestra total (evaluación de las diferencias entre individuos masculinos y femeninos); y, 2) Sexo por períodos Temprano, Medio y Tardío (evaluación de las diferencias entre sexos, entre períodos y de la interacción sexo por período). Se empleó la prueba del análisis de la varianza con covariantes, tanto desde el punto de vista univariado como multivariado (ANCOVA y MANCOVA) para uno o dos criterios de clasificación (Sokal y Rohlf 1979; Seber 1984). En el primer caso se usaron como covariantes: período, edad y deformación artificial. En el segundo caso: sexo por período, las covariantes fueron edad y deformación artificial. Este análisis permitió poner a prueba la posible interacción entre sexo y período para poder evaluar en forma independiente las variaciones del SD desde el punto de vista temporal. Se aplicó el procedimiento del análisis discriminante (DA) para comprobar la asignación del sexo de cada individuo realizada durante el relevamiento, mediante la reclasificación de las observaciones según la mayor probabilidad de pertenecer a uno u otro sexo (Seber 1984).

La magnitud del SD se midió por un índice de dimorfismo sexual (SDI), como la proporción de la diferencia entre la media de masculinos y la de femeninos con respecto a la media de femeninos (Garn et al. 1967). Su valor indica la sensibilidad ambiental de los individuos masculinos con respecto a los femeninos de acuerdo con Frayer y Wolpoff (1985) y permite efectuar comparaciones con la información disponible en la bibliografía sobre el tema

\begin{tabular}{|l|c|c|c|c|c|}
\hline \multicolumn{7}{|c|}{ Deformación artificial } \\
\hline \multicolumn{1}{|c|}{ Período } & Tabular erecta & Tabular oblicua & Circular & No deformado & Total \\
\hline Temprano & $13(30 \%)$ & $9(20 \%)$ & $2(5 \%)$ & $20(45 \%)$ & $44(100 \%)$ \\
\hline Medio & $206(43 \%)$ & $91(19 \%)$ & $29(6 \%)$ & $150(32 \%)$ & $476(100 \%)$ \\
\hline Tardío & $40(38 \%)$ & $27(26 \%)$ & $6(6 \%)$ & $31(30 \%)$ & $104(100 \%)$ \\
\hline Total & $\mathbf{2 5 9}(\mathbf{4 2} \%)$ & $\mathbf{1 2 7}(\mathbf{2 0} \%)$ & $\mathbf{3 7}(\mathbf{6} \%)$ & $\mathbf{2 0 1}(\mathbf{3 2} \%)$ & $\mathbf{6 2 4}(\mathbf{1 0 0} \%)$ \\
\hline
\end{tabular}

Tabla 3. Dimorfismo sexual: Distribución de la muestra por deformación artificial y por período. Nota: $\chi^{2}=6.895$, con $6 \mathrm{gl}$. y p=0.33064. 
(Howell 1973; Cocilovo 1978; Cocilovo et al. 1982; Cocilovo y Baffi 1985; Baffi y Cocilovo 1989; Cocilovo et al. 1994; Baffi et al. 1996; Cocilovo et al. 1999; L'Abbé et al. 2006; Kranioti et al. 2008; Cocilovo et al. 2013; Cocilovo y Varela 2014 Ms).

\section{$*$ Resultados}

La distribución de la muestra por período y por sitio se muestra en la Tabla 1. En el total de la serie, ambos sexos se encuentran repartidos de manera casi uniforme (51\% masculinos y $49 \%$ femeninos). Igualmente sucede con la distribución del género y del tipo deformatorio por período cultural (Tabla 2: $\chi^{2}=2.602$, con 2 gl. $y \mathrm{p}=0.27226 \mathrm{y}$ Tabla 3: $x^{2}=6.895$, con 6 gl. $y p=0.33064$ ).

En la Tabla 4 se exponen los resultados del ANCOVA (covariantes período, edad y deformación artificial) para la evaluación de las diferencias entre sexos en la muestra total. El análisis se presenta para cada variable individualmente y al pie figuran los resultados del análisis

\begin{tabular}{|c|c|c|c|}
\hline \multicolumn{4}{|c|}{ ANCOVA } \\
\hline Variables & $\mathrm{F}$ & $\mathrm{P}$ & SDI \\
\hline Longitud máxima (glabela) & 213.136 & $<0.0001$ & 0.044 \\
\hline Ancho máximo & $35 \cdot 475$ & $<0.0001$ & 0.019 \\
\hline Altura basilo-bregmática & 219.578 & $<0.0001$ & 0.042 \\
\hline Altura porio-bregmática & 169.774 & $<0.0001$ & 0.038 \\
\hline Diámetro frontal mínimo & 34.754 & $<0.0001$ & 0.022 \\
\hline Diámetro frontal máximo & 59.573 & $<0.0001$ & 0.028 \\
\hline Ancho fronto-malar & 207.958 & $<0.0001$ & 0.040 \\
\hline Ancho bimastoidea mínimo & 106.073 & $<0.0001$ & 0.039 \\
\hline Ancho bimastoidea máximo & 252.703 & $<0.0001$ & 0.049 \\
\hline Ancho bicigomático & 530408 & $<0.0001$ & 0.068 \\
\hline Ancho bimaxilar máximo & 172.588 & $<0.0001$ & 0.047 \\
\hline Ancho biastérico & 41.025 & $<0.0001$ & 0.024 \\
\hline Ancho biauricular & 208.516 & $<0.0001$ & 0.052 \\
\hline Diámetro nasio-basilar & 268.810 & $<0.0001$ & 0.053 \\
\hline Diámetro alvéolo-basilar & 121.969 & $<0.0001$ & 0.048 \\
\hline Altura nasio-alveolar & 121.320 & $<0.0001$ & 0.060 \\
\hline Altura de la nariz & 72.587 & $<0.0001$ & 0.038 \\
\hline Ancho de la nariz & 12.052 & $<0.0001$ & 0.019 \\
\hline Altura del pómulo & 124.502 & $<0.0001$ & 0.095 \\
\hline Distancia interorbitaria anterior & 54.776 & $<0.0001$ & 0.047 \\
\hline Ancho biorbitario & 150.510 & $<0.0001$ & 0.033 \\
\hline Ancho de la órbita & 53.946 & $<0.0001$ & 0.025 \\
\hline Altura de la órbita & 2.272 & 0.132 & -0.006 \\
\hline Ancho máxilo alveolar & 147.688 & $<0.0001$ & 0.073 \\
\hline Longitud máxilo alveolar & 126.808 & $<0.0001$ & 0.060 \\
\hline Longitud del paladar & 44.692 & $<0.0001$ & 0.036 \\
\hline Ancho del paladar & 0.100 & 0.752 & 0.002 \\
\hline Altura órbito alveolar & 98.359 & $<0.0001$ & 0.077 \\
\hline Longitud foramen magnum & 50.550 & $<0.0001$ & 0.034 \\
\hline Ancho foramen magnum & 35.666 & $<0.0001$ & 0.037 \\
\hline Curva sagital nasio-bregma & 97.140 & $<0.0001$ & 0.036 \\
\hline Curva sagital bregma-lambda & 68.221 & $<0.0001$ & 0.047 \\
\hline Curva sagital lambda-opistion & 21.998 & $<0.0001$ & 0.028 \\
\hline Curva transversal & 117.746 & $<0.0001$ & 0.031 \\
\hline Curva horizontal & 284.764 & $<0.0001$ & 0.037 \\
\hline
\end{tabular}

Tabla 4. Dimorfismo sexual: Muestra total, prueba de diferencias entre medias, valores F y p con 1 y 612 gl. Covariantes: Período, edad y deformación artificial. SDI: Proporción de la diferencia entre medias de ambos sexos con respecto a la media femenina. Nota: Prueba

MANCOVA, Lambda de Wilks: 0.417821; F: 23.16976, con 35 y 582 gl, p<o.0oo1. 
multivariado. En la última columna se consignan los valores del índice de dimorfismo sexual (SDI). Desde el punto de vista univariado, con independencia de los efectos período, edad y deformación artificial, la mayoría de las características métricas (94\%) presentan promedios diferentes entre uno y otro sexo, excepto la altura de la órbita y el ancho del paladar. El análisis conjunto (MANCOVA) para todas las variables también indica diferencias significativas entre ambos géneros. El índice medio de dimorfismo sexual fue de $0.041(s e=0.0034)$, el valor mínimo - 0.006 fue para la altura de la órbita y el máximo 0.095 para la altura del pómulo.

En la Tabla 5 figuran los resultados del análisis del ANCOVA sexo por período (covariantes edad y deformación artificial) para cada variable, y al pie los resultados del análisis multivariado. El $49 \%$ de las variables expresa diferencias significativas entre períodos y el $94 \%$ con respecto al sexo, mientras que no se registran pruebas de interacción entre los factores sexo y período. Nue-

\begin{tabular}{|c|c|c|c|c|c|c|}
\hline \multirow{3}{*}{$\begin{array}{c}\text { Efecto } \\
\text { Variable }\end{array}$} & \multicolumn{6}{|c|}{ ANCOVA } \\
\hline & \multicolumn{2}{|c|}{ Período } & \multicolumn{2}{|c|}{ Sexo } & \multicolumn{2}{|c|}{ Período por sexo } \\
\hline & $\mathrm{F}$ & $\mathrm{P}$ & $\mathrm{F}$ & $\mathrm{P}$ & $\mathrm{F}$ & $\mathrm{P}$ \\
\hline Longitud máxima (glabela) & 0.49 & 0.6098 & 84.47 & $<0.0001$ & 1.54 & 0.2155 \\
\hline Ancho máximo & 15.26 & $<0.0001$ & 20.24 & $<0.0001$ & 0.41 & 0.6666 \\
\hline Altura basilo-bregmática & 3.34 & 0.0361 & 87.19 & $<0.0001$ & 0.25 & 0.7794 \\
\hline Altura porio-bregmática & 1.87 & 0.1557 & 89.8 & $<0.0001$ & 1.69 & 0.1855 \\
\hline Diámetro frontal mínimo & 1.943 & 0.1441 & 17.261 & $<0.0001$ & 0.392 & 0.6757 \\
\hline Diámetro frontal máximo & 7.534 & 0.0006 & $35 \cdot 345$ & $<0.0001$ & 0.592 & 0.5536 \\
\hline Ancho fronto-malar & 6.46 & 0.0017 & 90.31 & $<0.0001$ & 0.2 & 0.8167 \\
\hline Ancho bimastoidea mínimo & 15.027 & $<0.0001$ & 39.79 & $<0.0001$ & 1.408 & 0.2454 \\
\hline Ancho bimastoidea máximo & 4.6 & 0.0104 & 100.26 & $<0.0001$ & 1.39 & 0.2505 \\
\hline Ancho bicigomático & 11.28 & $<0.0001$ & 181.55 & $<0.0001$ & 1.58 & 0.2058 \\
\hline Ancho bimaxilar máximo & 0.537 & 0.5846 & 76.772 & $<0.0001$ & 0.114 & 0.8922 \\
\hline Ancho biastérico & 15.367 & $<0.0001$ & 19.026 & $<0.0001$ & 0.356 & 0.7003 \\
\hline Ancho biauricular & 11.48 & $<0.0001$ & 77.83 & $<0.0001$ & 2.6 & 0.0749 \\
\hline Diámetro nasio-basilar & 6.65 & 0.0014 & 101.82 & $<0.0001$ & 0.81 & 0.4473 \\
\hline Diámetro alvéolo-basilar & 1.456 & 0.2340 & 49.408 & $<0.0001$ & 0.004 & 0.9956 \\
\hline Altura nasio-alveolar & 2.527 & 0.0807 & 50.565 & $<0.0001$ & 0.176 & 0.8385 \\
\hline Altura de la nariz & 5.291 & 0.0053 & 38.355 & $<0.0001$ & 1.96 & 0.1418 \\
\hline Ancho de la nariz & 1.059 & 0.3475 & 14.707 & $<0.0001$ & 2.266 & 0.1046 \\
\hline Altura del pómulo & 3.577 & 0.0286 & 56.141 & $<0.0001$ & 0.154 & 0.8573 \\
\hline Distancia interorbitaria anterior & 1.776 & 0.1702 & 20.016 & $<0.0001$ & 0.875 & 0.4173 \\
\hline Ancho biorbitario & 5.42 & 0.0046 & 58.95 & $<0.0001$ & 0.36 & 0.6958 \\
\hline Ancho de la órbita & 1.728 & 0.1784 & 21.215 & $<0.0001$ & 0.289 & 0.7493 \\
\hline Altura de la órbita & 10.326 & $<0.0001$ & 0.038 & 0.8459 & 0.728 & 0.4835 \\
\hline Ancho maxilo alveolar & 0.64 & 0.5275 & 51.792 & $<0.0001$ & 0.829 & 0.4370 \\
\hline Longitud máxilo alveolar & 0.832 & 0.4358 & $45 \cdot 408$ & $<0.0001$ & 0.287 & 0.7507 \\
\hline Longitud del paladar & 2.993 & 0.0509 & 16.64 & 0.0001 & 1.58 & 0.2068 \\
\hline Ancho del paladar & 0.434 & 0.6480 & 0.424 & 0.5151 & 0.529 & 0.5894 \\
\hline Altura órbito alveolar & 0.728 & 0.4833 & 39.779 & $<0.0001$ & ool1 & 0.9894 \\
\hline Longitud foramen magnum & 1.292 & 0.2756 & 31.129 & $<0.0001$ & 0845 & 0.4302 \\
\hline Ancho foramen magnum & 6.824 & 0.0012 & 15.072 & 0.0001 & 0.017 & 0.9833 \\
\hline Curva sagital nasio-bregma & 5.569 & 0.0040 & 38.407 & $<0.0001$ & 0.074 & 0.9288 \\
\hline Curva sagital bregma-lambda & 1.085 & 0.3384 & 36.047 & $<0.0001$ & 0384 & 0.6812 \\
\hline Curva sagital lambda-opistion & 0.336 & 0.7145 & 9.145 & 0.0026 & 2.062 & 0.1281 \\
\hline Curva transversal & 0.51 & 0.5992 & 57.97 & $<0.0001$ & 0.65 & 0.5199 \\
\hline Curva horizontal & 7.86 & 0.0004 & 121.7 & $<0.0001$ & 2.34 & 0.0967 \\
\hline GL & 2 & & 1 & & 2 & \\
\hline
\end{tabular}

Tabla 5. Dimorfismo sexual: Sexo por período, prueba de diferencias entre medias, valores F y P. Covariantes: Edad y deformación artificial. Nota: Prueba ANCOVA para diferencias de las medias entre período, sexos e interacción; grados de libertad del numerador en la última fila y grados de libertad del error es 613 para todos los casos. Prueba MANCOVA, Período: Lambda de Wilks: 0.654356, F: 3.908, gl: 70 y 1158, p<0.0001; Sexo: Lambda de Wilks: 0.641239, F: 9.255, gl: 35 y 579, p<0.00o1; Período por sexo: Lambda de Wilks: 0.88878 , F: $1.005, \mathrm{gl}: 70$ y $1158, \mathrm{p}=0.46902$. 
vamente la altura de la órbita y el ancho del paladar no manifiestan dimorfismo sexual. Desde el punto de vista multivariado las diferencias fenotípicas entre sexos y entre períodos son confirmadas, no existiendo pruebas suficientes para poner en evidencia el efecto interacción sexo por período. Los valores SDI para cada período se presentan en la Tabla 6, y al pie, la media de cada uno junto con el error estándar (SE) y los valores máximos y mínimos. No se observan diferencias marcadas de este indicador entre los períodos. Los valores mínimos se vinculan con la curva lambda opistion y la altura de la órbita y los máximos con la altura del pómulo. Las Figuras 2, 3 y 4 grafican la distribución de los valores medios $( \pm \mathrm{SE})$

\begin{tabular}{|c|c|c|c|}
\hline \multirow[b]{2}{*}{ Variables } & \multicolumn{3}{|c|}{ DSI } \\
\hline & Temprano & Medio & Tardío \\
\hline Longitud máxima (glabela) & 0.033 & 0.043 & 0.055 \\
\hline Ancho máximo & 0.022 & 0.018 & 0.026 \\
\hline Altura basilo-bregmática & 0.044 & 0.043 & 0.037 \\
\hline Altura porio-bregmática & 0.058 & 0.037 & 0.035 \\
\hline Diámetro frontal mínimo & 0.033 & 0.022 & 0.018 \\
\hline Diámetro frontal máximo & 0.042 & 0.027 & 0.031 \\
\hline Ancho fronto-malar & 0.039 & 0.039 & 0.044 \\
\hline Ancho bimastoidea mínimo & 0.021 & 0.040 & 0.049 \\
\hline Ancho bimastoidea máximo & 0.037 & 0.049 & 0.060 \\
\hline Ancho bicigomático & 0.052 & 0.071 & 0.063 \\
\hline Ancho bimaxilar máximo & 0.051 & 0.046 & 0.050 \\
\hline Ancho biastérico & 0.020 & 0.024 & 0.032 \\
\hline Ancho biauricular & 0.028 & 0.052 & 0.065 \\
\hline Diámetro nasio-basilar & 0.054 & 0.055 & 0.044 \\
\hline Diámetro alvéolo-basilar & 0.046 & 0.048 & 0.049 \\
\hline Altura nasio-alveolar & 0.068 & 0.060 & 0.053 \\
\hline Altura de la nariz & 0.067 & 0.038 & 0.025 \\
\hline Ancho de la nariz & 0.060 & 0.014 & 0.028 \\
\hline Altura del pómulo & 0.110 & 0.092 & 0.095 \\
\hline Distancia interorbitaria anterior & 0.054 & 0.051 & 0.029 \\
\hline Ancho biorbitario & 0.026 & 0.033 & 0.037 \\
\hline Ancho de la órbita & 0.019 & 0.025 & 0.030 \\
\hline Altura de la órbita & 0.013 & -0.007 & -0.009 \\
\hline Ancho máxilo alveolar & 0.046 & 0.073 & 0.083 \\
\hline Longitud máxilo alveolar & 0.045 & 0.060 & 0.063 \\
\hline Longitud del paladar & 0.014 & 0.035 & 0.056 \\
\hline Ancho del paladar & 0.024 & 0.002 & -0.006 \\
\hline Altura órbito alveolar & 0.079 & 0.078 & 0.074 \\
\hline Longitud foramen magnum & 0.054 & 0.031 & 0.042 \\
\hline Ancho foramen magnum & 0.038 & 0.039 & 0.037 \\
\hline Curva sagital nasio-bregma & 0.037 & 0.036 & 0.032 \\
\hline Curva sagital bregma-lambda & 0.064 & 0.045 & 0.051 \\
\hline Curva sagital lambda-opistion & 0.007 & 0.024 & 0.053 \\
\hline Curva transversal & 0.043 & 0.030 & 0.029 \\
\hline Curva horizontal & 0.029 & 0.036 & 0.048 \\
\hline $\mathrm{N}$ & 35 & 35 & 35 \\
\hline Media & 0.042 & 0.040 & 0.043 \\
\hline SE & 0.004 & 0.003 & 0.004 \\
\hline Mínimo & $0.007^{1}$ & $-0.007^{3}$ & $-0.009^{5}$ \\
\hline Máximo & $0.110^{2}$ & $0.092^{4}$ & $0.095^{6}$ \\
\hline
\end{tabular}

Tabla 6. Índice de dimorfismo sexual por período (SDI). Nota: ${ }^{1}$ Curva lambda opistion, ${ }^{2,4,6}$ altura del pómulo, ${ }^{3,5}$ altura de la órbita.

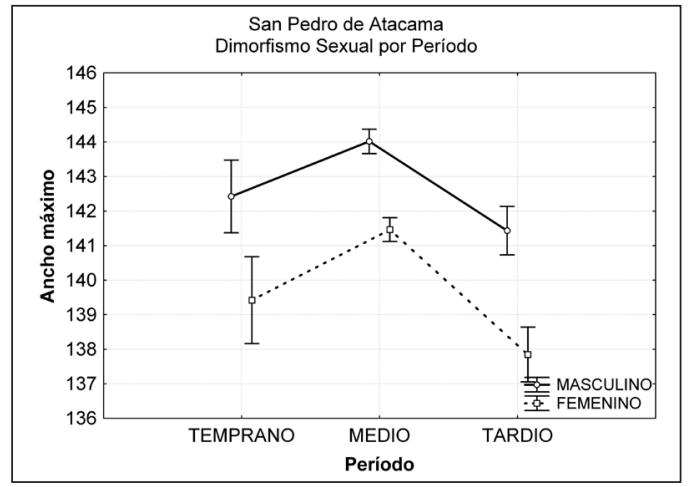

Figura 2. Ancho máximo y dimorfismo sexual por período. Se presentan los valores medios y el error estándar en las barras verticales $( \pm)$.

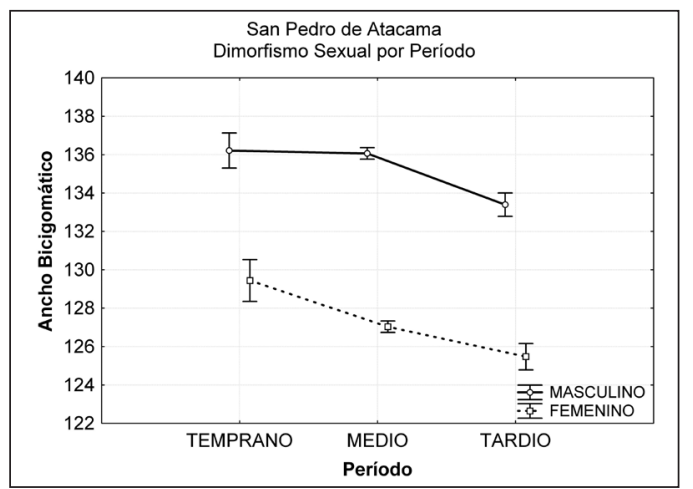

Figura 3. Ancho bicigomático y dimorfismo sexual por período. Se presentan los valores medios y el error estándar en las barras verticales $( \pm)$.

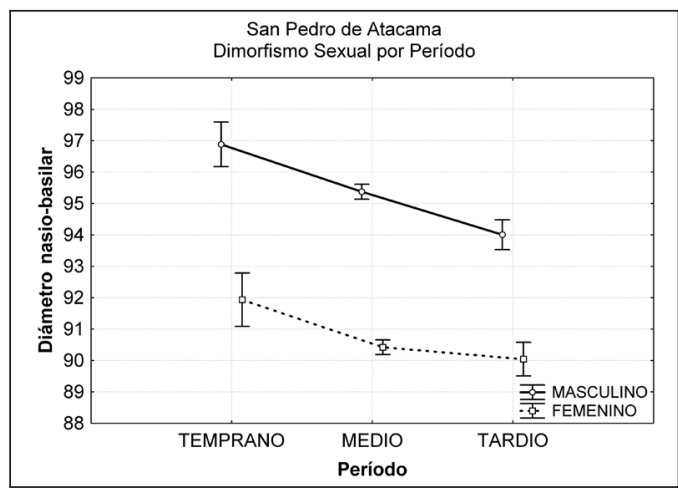

Figura 4. Diámetro nasio-basilar y dimorfismo sexual por período. Se presentan los valores medios y el error estándar en las barras verticales $( \pm)$. 
por sexo para cada período de las variables: ancho máximo del cráneo, ancho bicigomático y diámetro nasiobasilar. Las diferencias entre ambos sexos se expresan claramente en los tres períodos, igualmente se observan fluctuaciones marcadas y paralelas en el fenotipo medio de cada sexo por período.

En la Tabla 7 se observan los resultados de la reclasificación de las observaciones por medio de la función discriminante, y al pie de la misma, la prueba multivariada (MANCOVA) sobre las medias de los sexos indicando diferencias significativas entre los fenotipos medios. Se destaca la asignación del $88.4 \%$ de los individuos al sexo correcto. La Figura 5 muestra la distribución de los individuos por sexo de acuerdo con la primera coordenada discriminante canónica que acumula el 100\% de la varianza total. Las Figuras 6 y 7 muestran cráneos masculinos y femeninos de los sitios Coyo 3 y Quitor 5.

\section{* Discusión Y CONClusiones}

De acuerdo con los resultados expuestos, la distribución uniforme de los individuos según el sexo y por período fortalece las distintas pruebas estadísticas realizadas sobre las diferencias morfológicas entre individuos masculinos y femeninos en la muestra total, y sobre su variación con respecto al período cultural. Por otra parte, los diseños de las pruebas ANCOVA y MANCOVA pueden ser aceptadas con suficiente confianza no solo por los grados de libertad disponibles, sino también por su independencia de otros factores de variación incluidos en el modelo como covariantes: edad, deformación artificial y período en el primer caso, y edad y deformación artificial en el segundo caso.
La variación de los fenotipos medios por período ( $\mathrm{Ta}-$ bla 4) probado tanto desde el punto de vista univariado como multivariado y ejemplificado para algunas variables (ver Figuras 2, 3 y 4) revela un proceso microevolutivo particular de la localidad que involucró a ambos sexos en forma independiente de las diferencias entre ellos. Las características principales y las causas de este fenómeno atribuido a los efectos combinados de la deriva y la migración han sido destacadas en trabajos anteriores (Varela y Cocilovo 1996, 2000, 2002, 2009; Varela 1997).

La reclasificación de los individuos por medio del análisis discriminante logra el $88.4 \%$ de acierto es un valor aceptable para la determinación del sexo basado en atributos exclusivamente del cráneo y es coherente con experiencias anteriores para la región (Varela et al. 1990; Cocilovo et al. 2013; Cocilovo y Varela $2014 \mathrm{Ms}$ ).

En la muestra total la expresión del SD en la mayoría de las estructuras funcionales del cráneo es completa, con la excepción de dos variables (altura de la órbita y ancho del paladar), en las cuales se refleja el más bajo valor de SDI, un hecho que había sido probado anteriormente en esta localidad (Varela et al. 1990) y que se repite en Pisagua (Cocilovo et al. 1999), Coyo Oriental (Cocilovo et al. 1994), y en Arica solo en la primera variable (Cocilovo y Varela $2014 \mathrm{Ms}$ ); en los restantes casos los valores de este indicador son positivos indicando mayores dimensiones en individuos masculinos con respecto a femeninos. Las variables más dimórficas son: altura del pómulo, ancho máxilo-alveolar y bicigomático. En el diseño sexo por período, los resultados son semejantes.

Si bien se pusieron de manifiesto las diferencias entre períodos, el componente más importante de la variación

\begin{tabular}{|c|c|c|c|c|}
\hline & & \multicolumn{2}{|c|}{ Reasignación } & \multirow{3}{*}{ Total } \\
\hline \multicolumn{2}{|c|}{ Casos asignados al sexo correcto } & Masculino & Femenino & \\
\hline Sexo & $\%$ & $p=0.51208$ & $\mathrm{p}=0.48792$ & \\
\hline Masculino & 85.9 & 274 & 45 & 319 \\
\hline Femenino & 91.1 & 27 & 276 & 303 \\
\hline Total & 88.4 & 301 & 321 & 622 \\
\hline
\end{tabular}

Tabla 7. Reclasificación de las observaciones por medio de la función discriminante. Nota: Prueba de diferencia entre medias: Lambda de Wilks: 0.42233 aprox, $F_{(12.608)}=69.303 ;$ p $<0.0001$. 
total es el SD cuya expresión es independiente de ese factor, hecho que fue también probado en Arica (Cocilovo y Varela 2014 Ms). En los tres períodos de San Pedro de Atacama las variables más expresivas con respecto al SD son: altura del pómulo y altura órbito-alveolar; se suman en el período Temprano, altura nasio-alveolar y altura de la nariz; en el período Medio, ancho bicigomático y

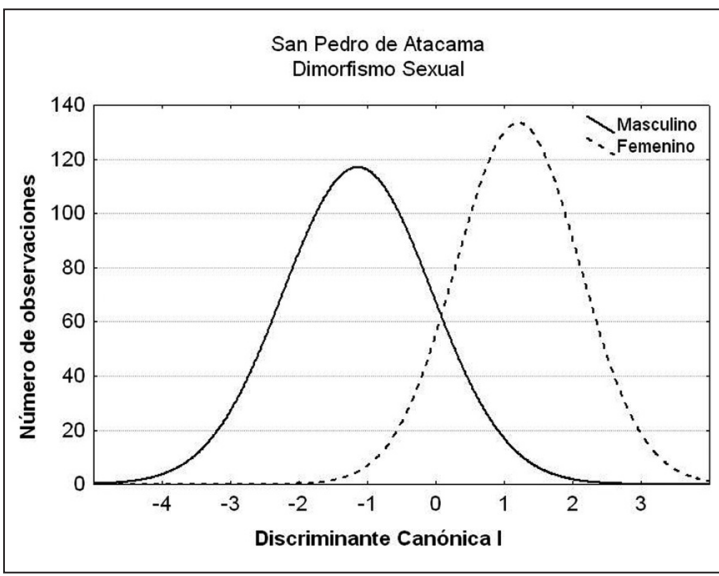

Figura 5. Distribución de ambos sexos en la muestra total de acuerdo con la primera variable discriminante canónica.

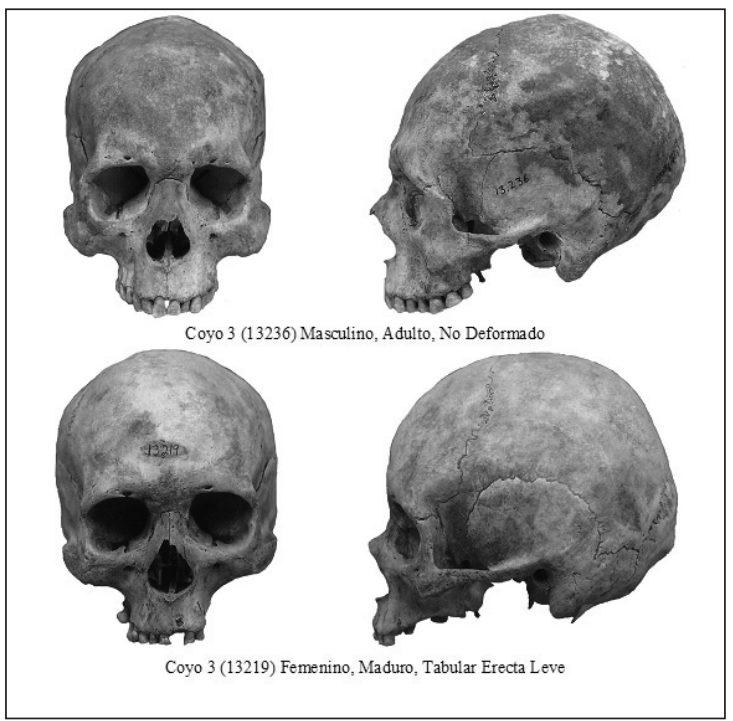

Figura 6. Coyo 3 , individuos masculino, adulto, no deformado (Catálogo: 13236) y femenino, maduro, tabular erecta leve (Catálogo: 13219). maxilo-alveolar, ambas se repiten en el período Tardío y se agrega en éste, el ancho biauricular.

Desde el punto de vista multivariado, la diferencia entre los vectores medios de ambos géneros está claramente demostrada en la muestra total (Lambda de Wilks: 0.417821 ; F: 23.1698, con 35 y 582 gl, p<0.0001). La expresión global del SDI fue de $4.1 \%(\mathrm{SE}=0.34 \%)$ valor que se mantiene constante entre períodos. Igualmente se pudo comprobar en el diseño período por sexo ( $\mathrm{Pe}$ ríodo: Lambda de Wilks: 0.654356, F: 3.908, gl: 70 y 1158, p<0.0001; Sexo: Lambda de Wilks: 0.641239, F: 9.255, gl: 35 y 579, p<0.0001; Período por sexo: Lambda de Wilks: 0.88878 , F: 1.005, gl: 70 y 1158 , p=0.46902) en donde se destaca la significación estadística de ambos efectos principales y la ausencia de interacción entre ellos; es decir, por este último hecho, ambos factores se expresan independientemente y no se observan cambios en la expresión de SD en el tiempo. De hecho, en la Tabla 5 puede observarse que los valores medios por período del SDI son semejantes, con un muy ligero descenso en el período Medio.

Para varias localidades del área Centro-Sur Andina se dispone de mediciones del SDI que permiten evaluar

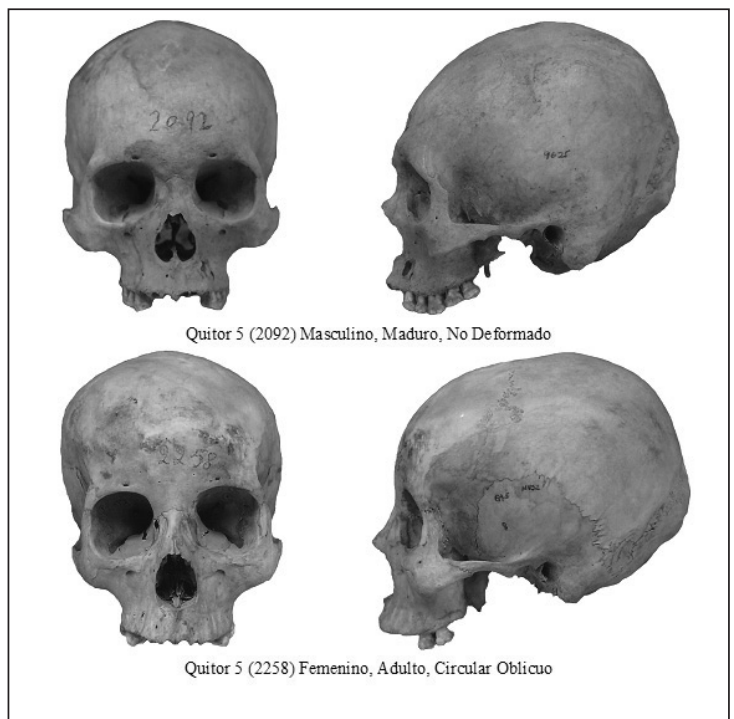

Figura 7. Quitor 5, individuos masculino, maduro, no deformado (Catálogo: 2092) y femenino, adulto, circular oblicuo (Catálogo: 2258). 
más objetivamente los resultados obtenidos en este trabajo. En Cochabamba (Bolivia) se obtuvo un registro de $0.0490 \pm 0.0062$ a partir de 31 variables (Cocilovo et al. 2013). En Arica el índice SDI fue 0.0394 $\pm 0.0027(3000$ AC-1450 DC), en Pisagua: $0.0363 \pm 0.0046$ (300 AC1450 DC), en Caleta Huelén 42: $0.042 \pm 0.005$ (2000 AC), Punta Teatinos: $0.0416 \pm 0.0055$ (3000 AC) y El Cerrito: $0.0426 \pm 0.0040$ (2000 AC) (Cocilovo y Varela 2014 Ms). En el Noroeste Argentino para Pampa Grande (Las Pirguas: 600 DC) con 39 variables, la medición de este rasgo fue de $0.027 \pm 0.0035$, lo cual marca el valor mínimo a escala regional, indicando una baja calidad de vida en condiciones extremas de subsistencia (Baffi y Cocilovo 1989; Baffi et al. 1996). Por otra parte, utilizando los datos publicados de casos similares, se estimaron valores SDI en mediciones craneanas con fines comparativos: Creta, grupos actuales (s. XIX-XX): $0.0570 \pm 0.0060$ (Kranioti et al. 2008), Sudáfrica, Ven$\mathrm{da}($ s. XX): $0.0212 \pm 0.0053$ (L'Abbé et al. 2006) y a nivel mundial: $0.064 \pm 0.008$, a partir de la base de datos de Howell (1973) con 80 variables y una muestra de más de 30 localidades.

De acuerdo con los datos existentes a nivel regional y extra regional y a las pruebas estadísticas realizadas en la población atacameña prehistórica, se observa un proceso de crecimiento y desarrollo diferencial de ambos géneros adecuado a las condiciones locales y en un entorno ambiental y social con un impacto moderado en la calidad de vida de la población. Los resultados obtenidos y el valor medio SDI de 0.041 (SE = 0.0034) no difieren substancialmente de los registros realizados para otras localidades del norte de Chile, y presenta un valor intermedio entre los límites antes expuestos.

La comparación de la información obtenida en este trabajo con aquella disponible sobre indicadores de estrés basados en atributos sobre patologías y traumatismos, no es fácil por cuestiones vinculadas con la escala del diseño y la carencia de ese registro en varios de los sitios que integran la muestra aquí empleada. Además, las consideraciones realizadas por Luna (2006) sobre los alcances y limitaciones de las muestras osteológicas para realizar inferencias acerca de la población que las generó, brinda un marco de referencia apropiado sobre las posibles coincidencias y discrepancias al intentar relacionar ambas fuentes de datos.
En San Pedro de Atacama, la calidad de vida y estabilidad de la población dependieron de la disponibilidad y distribución de los recursos y de la magnitud de la interacción con otras áreas. En efecto, los estudios detectaron momentos de paz y prosperidad alternando con otros donde prevalecieron condiciones menos propicias. En líneas generales, los indicadores de violencia y de impacto ambiental fueron menos frecuentes en los períodos Intermedio Temprano (Toconao Oriente: 200 AC-600 DC), Medio (Solcor 3: 600-950 DC) y en el Tardío (Catarpe 1-2: 1400-1532 DC), comparados con los registros del período Intermedio Tardío (sitios Quitor 6 Tardío, Coyo 3, Yaye y Catarpe 4-5) (Costa 1988; Costa y Llagostera 1994; Costa et al. 2004; Torres-Rouff et al. 2005; Torres-Rouff y Costa 2006; Torres-Rouff 2011). En Solcor 3 los indicadores de violencia alcanzaron un 25\% (datos promediados de Lessa 1999) y para Coyo Oriental un $11.06 \%$ (Lessa y Mendonça 2007). Si bien nuestro actual diseño cumplió con el objetivo propuesto, los resultados no acompañan las expectativas generadas por los trabajos antes citados, teniendo en cuenta que la ausencia de interacción sexo por período, implica que las variaciones en la expresión del SD entre los períodos consideradas (Temprano, Medio y Tardío) son mínimas, lo cual puede ser observado gráficamente en las Figuras 2, 3 y 4.

Aunque el nivel de solapamiento entre la información disponible sobre la calidad de vida evaluada a partir de marcadores relacionados con situaciones de violencia, nutrición e infecciones y la expresión del SD obtenida en este trabajo parezca irreconciliable, es posible que la asociación entre ambas fuentes de datos pueda ser probada en algunas situaciones extremas, por ejemplo, cuando se encuentra comprometida la propia subsistencia del grupo como en Las Pirguas (Baffi y Cocilovo 1989; Baffi et al. 1996) y en otras sea más difícil de comprobar cuando prevalecen condiciones de vida menos rigurosas. En tal sentido, es apropiada la observación realizada por Costa y colaboradores (2004) acerca de una mejor calidad de vida en Solcor 3, durante la influencia de Tiwanaku (400-900 DC), a partir de un incremento de SD en la talla, sin asociación con la variación de otros indicadores como la hiperostosis porótica y la hipoplasia del esmalte dental, o con la mayor frecuencia de periostitis y osteomielitis, como fue advertido también por $\mathrm{Da}$ Gloria y colaboradores (2011). 
Por otra parte, se puede pensar que el crecimiento y desarrollo del cráneo al alcanzar el valor adulto más temprano que el resto del esqueleto fue menos sensible al impacto de factores ambientales adversos. Por ejemplo, tomando como referencia general la información obtenida en grupos actuales y salvando las diferencias entre las muestras, a los 10 años de edad, las dimensiones de la bóveda craneal alcanzan entre un 95 y 97\%, la altura de la órbita un 97\%, y las dimensiones de la cara entre un 84 y $87 \%$ con relación al valor adulto (Scott 1953 , 1958). Además, se registran cambios menores hasta la edad adulta en la longitud y altura de la pirámide facial (West y McNamara 1999). A partir de una muestra de individuos de clase media superior (Denver, Colorado, EE.UU.), Buschang y colaboradores (1983) presentan valores de maduración a los 15.5 años (con respecto al valor adulto), de $96 \%$ para la talla y entre un $94 \%$ y $100 \%$ para diversas dimensiones de la bóveda craneal y facial. Aunque tales relaciones no puedan ser generalizadas a otras poblaciones, sirven para explicar provisoriamente la magnitud y la estabilidad del SD observado a partir de mediciones craneanas no solo en San Pedro de Atacama sino también en varios sitios del norte de Chile que hemos citado.

Por último, destacamos que nuestro registro es más amplio y diverso, permitiendo resultados globales más estables y próximos al promedio regional. En todo caso, es preciso remarcar que la información obtenida en este trabajo no permite inferir cambios notables en la calidad de vida como para producir un impacto demostrable en el crecimiento y desarrollo normal del cráneo en la antigua población de San Pedro de Atacama. Además, esta conclusión no puede ser generalizada al resto del esqueleto, y tampoco implica que las diferencias en las condiciones de vida entre grupos contemporáneos y entre momentos distintos de la historia de la población no hayan influido en el crecimiento y desarrollo normal de los individuos con consecuencias en el SD, pues un estudio de mayor resolución, contando con muestras suficientes en cada nivel de los distintos factores ensayados (sexo por período, sexo por fase y sexo por sitio) podría en el futuro ponerlo de manifiesto.

Agradecimientos La realización de este trabajo fue posible gracias a la colaboración desinteresada de personas que en muchas oportunidades nos acompañaron y permitieron el desarrollo de nuestra tarea en un ambiente realmente confortable. Mencionamos en primer lugar a María Antonietta Costa y a Agustín Llagostera, a Macarena Oviedo y al personal del Instituto de Investigaciones Arqueológicas y Museo R.P. Gustavo Le Paige de la Universidad Católica del Norte. Nuestro especial recuerdo para Ulises Cárdenas y su familia, en particular su abuela Gumersinda Titichoca por su ayuda y cálida amistad. También al Dr. Julián Valetti por su ayuda en la preparación de las figuras, y al Dr. Marcelo Arana por la corrección del Abstract.

\section{* Referencias citadas}

AGÜERO, C., 2005. Aproximación al asentamiento humano temprano en los oasis de San Pedro de Atacama. Estudios Atacameños 30: $29-60$.

BAFFI, E. y J. COCILOVO, 1989. Evaluación del impacto ambiental en una población prehistórica: El caso de Las Pirguas (Salta, Argentina). Revista de Antropología 4: 39-43.

BAFFI, E., M. TORRES y J. COCILOVO, 1996. La población prehispánica de Las Pirguas (Salta, Argentina). Un enfoque integral. Revista Argentina de Antropología Biológica 1 (1): 204-218.

BERENGUeR, J., A. DEZA, A. ROMÁN y A. LlagOSTERA, 1986. La secuencia de Myriam Tarragó para San Pedro de Ata- cama: Un test por termoluminiscencia. Revista Chilena de Antropología 5: 17-54.

BINFORD, M., A. KOLATA, M. BRENNER, J. JANUSEK, M. SEDDON, M. ABBOTT y J. CURTIS, 1997. Climate variation and the rise and fall of an Andean civilization. Quaternary Research 47: 235-248.

BRAVO, L. y A. LLAGOSTERA, 1986. Solcor 3: Un aporte al conocimiento de la Cultura San Pedro. Período 500 al 900 DC. Chungara 16-17: 323-332.

BUIKSTRA, J. y D. UBELAKER, 1994. Standards for data collection from human skeletal remains. Arkansas Archaeological Survey, Fayetteville. 
BUSCHANG, P., R. BAUME y G. NASS, 1983. A craniofacial growth maturity gradient for males and females between four and 16 years of age. American Journal of Physical Anthropology 61 (3): 373-381.

COCILOVO, J., 1975. Estudio de dos factores que influencian la morfología craneana en una colección andina: El sexo y la deformación artificial. Revista Instituto de Antropología, $3^{\mathrm{a}}$ serie, 2 : 197-212.

1978. Estudio de dos factores que influyen en la morfología craneana en una colección patagónica: El sexo y la deformación artificial. Arquivos de Anatomía e Arqueología 3: 113-141.

COCILOVO, J. y E. BAFFI, 1985. Contribución al conocimiento de las características biológicas de la población prehistórica de Puerta de La Paya (Salta). Runa 15: 153-178.

COCILOVO, J. y H. VARELA, 2014 Ms. Las cuestiones de género en la población prehispánica de Arica: Dimorfismo sexual y calidad de vida.

COCILOVO J., F. ROTHHAMMER, S. QUEVEDO y E. LLOP, 1982. Microevolución en poblaciones prehistóricas del Área Andina. 3. La población del Morro de Arica. Craneometría. Revista de la Universidad Nacional de Río Cuarto 2: 91-111.

COCILOVO J., M. ZAVATTIERI y M. A. COSTA, 1994. Biología del grupo prehistórico de Coyo Oriental (San Pedro de Atacama, norte de Chile): I Dimorfismo sexual y variación etaria. Estudios Atacameños 11: 121-133.

COCILOVO, J., S. QUEVEDO, H. VARELA, S. VALDANO y M. CASTRO, 1999. Biología del grupo prehistórico de Pisagua, costa norte de Chile. Estudios Atacameños 17: 207-235.

COCILOVO, J., H. VARELA, M. A. COSTA y S. QUEVEDO, 2005. Los pescadores arcaicos de la desembocadura del río Loa (norte de Chile): El sitio Caleta Huelén 42. Chungara, Revista de Antropología Chilena 37 (1): 5-19.

COCILOVO, J., M. FUCHS, T. O'BRIEN y H. VARELA, 2013. Sexual dimorphism in prehispanic populations of the Cochabamba Valleys, Bolivia. Advances in Anthropology 3 (1): 10-15. http:// www.SciRP.org/journal/aa. DOI:10.4236/aa.2012.

COSTA, M. A., 1988. Reconstitución física y cultural de la población tardía del cementerio Quitor 6 (San Pedro de Atacama). Estudios Atacameños 9: 99-126.

COSTA, M. A. y A. LLAGOSTERA, 1994. Coyo 3: Momentos finales del período Medio en San Pedro de Atacama. Estudios Atacameños 11: 73-107.
COSTA, M. A., W. NEVES, A. M. DE BARROS y R. BARTOLOMUCCI, 1999. Trauma y estrés en poblaciones prehistóricas de San Pedro de Atacama, norte de Chile. Chungara 30 (1): 65-74.

COSTA, M. A., W. NEVES y M. HUBBE, 2004. Influencia de Tiwanaku en la calidad de vida biológica de la población prehistórica de San Pedro de Atacama. Estudios Atacameños 27: 103-116.

COSTA, M. A., A. LlaGOSTERA y J. COCILOVO, 2008. La deformación craneana en la población prehistórica de Coyo Oriente, San Pedro de Atacama. Estudios Atacameños, Arqueología yAntropología Surandinas 36: 29-41.

DA GLORIA, P., W. NEVES, M. A. COSTA y R. BARTOLOMUC$\mathrm{CI}, 2011$. Nonspecific infectious diseases in prehistoric San Pedro de Atacama, northern Chile. Chungara, Revista de Antropología Chilena 43 (1): 135-146.

DEMBO, A. y J. IMBELLONI, 1938. Deformaciones intencionales del cuerpo humano. Humanior. Sección A3. Imprenta Luis L. Gotelli, Buenos Aires.

DETTWYLER, K., 1992. Nutritional status of adults in rural Mali. American Journal of Physical Anthropology 88 (3): 309-321.

FRAYER, D. y M. WOLPOFF, 1985. Sexual dimorphism. Annual Review of Anthropology 14 (1): 429-473.

GARN, S., A. LEWIS, D. SWINDLER y R. KEREWSKY, 1967. Genetic control of sexual dimorphism in tooth size. Journal of Dental Research 46: 963-972. http://jdr.sagepub.com

GENOVÉS, S., 1962. Introducción al diagnóstico de la edady del sexo en restos óseos prehistóricos. Universidad Nacional Autónoma de México, México D.F.

HOWELL, W., 1973. Cranial variation in man. Papers of the Peabody Museum of Archaeology and Ethnology 67. Harvard University, Cambridge.

HUbBE, M., C. TORRES-ROUFF, W. NEVES, L. KING, P. DA GLORIA y M. A. COSTA, 2012. Dental health in northern Chile's Atacama oases: Evaluating the Middle Horizon (AD 500-1000) impact on local diet. American Journal of Physical Anthropology 148 (1): 62-72.

KELLEY, J. y J. ANGEL, 1987. Life stresses of slavery. American Journal of Physical Anthropology 74 (2): 199-211.

KRANIOTI, E., M. ISCAN y M. MICHALODIMITRAKIS, 2008. Craniometric analysis of the modern Cretan population. Forensic Science International 180 (2-3): 110.e1-110.e5. DOI: 10.1016/j. forsciint.2008.06. 018. Epub. 
L'ABBÉ, E., I. RIBOT y M. STEYNI, 2006. A craniometric study of the $20^{\text {th }}$ Century Venda. South African Archaeological Bulletin 61 (183): $19-25$.

LE PAIGE, G., 1963. Continuidad y discontinuidad de la cultura atacameña. Anales de la Universidad del Norte 2: 5-25.

LESSA, A., 1999. Estudo de lesões traumáticas agudas como indicadores de tensão social na população do sítio-cemitério Solcor 3, San Pedro de Atacama, Chile. Disertación de Maestría en Salud Pública, Fundación Osvaldo Cruz, Río de Janeiro.

LESSA, A. y S. MENDONÇA, 2007. Gestación de un nuevo panorama social en el oasis atacameño: Conflictos durante la transición para el período de las Autonomías Regionales. Chungara, Revista de Antropología Chilena 39 (2): 209-220.

LLAGOSTERA, A. y M. A. COSTA, 1999. Patrones de asentamiento en la época agroalfarera de San Pedro de Atacama (norte de Chile). Estudios Atacameños 17: 175-206.

LlagosterA, A., C. TORRES y M. A. COSTA, 1988. El complejo psicotrópico en Solcor 3 (San Pedro de Atacama). Estudios Atacameños 9: 61-98.

LUNA, L., 2006, Alcances y limitaciones del concepto de estrés en bioarqueología. Antípoda 3: 255-279.

NADO, K., S. MARSTELLER, L. KING, B. DAVERMAN, C. TORRES-ROUFF y K. KNUDSON, 2012. Examining local social identities through patterns of biological and cultural variation in the Solcor ayllu, San Pedro de Atacama, Chile. Chungara, Revista de Antropología Chilena 44 (2): 341-357.

OAKLAND, A., 1992. Textiles and ethnicity: Tiwanaku in San Pedro de Atacama, north Chile. Latin American Antiquity 3 (4): $316-$ 340.

1994. Tradición e innovación en la prehistoria andina de San Pedro de Atacama. Estudios Atacameños 11: 109-120.

PÉREZ, B., G. RAMÍREZ, M. LANDAETA-JIMÉNEZ y M. VÁSQUEZ, 2010. Iconografía del dimorfismo sexual en dimensiones corporales y proporcionalidad, según estado nutricional en niños. El Hatillo, Caracas. Anales Venezolanos de Nutrición 23 (1): 10-17.

PURKAIT, R. y H. CHANDRA, 2002. Sexual dimorphism in Femora. Indian Study Forensic Science Communications Research and Technology 4 (3), www2.fbi.gov/hq/lab/fsc/backissu/julyzoo2/ purkait.htm.

2004. A study of sexual variation in Indian femur. Forensic Science International 146 (1): 25-33.
SCOTT, J., 1953. The growth of the human face. Proceedings of the Royal Society of Medicine 47 (2): 91-100.

1958. Further studies on the growth of the human face. Proceedings of the Royal Society of Medicine 52 (4):263-268.

SEBER, G., 1984. Multivariate observations. John Wiley \& Sons, Nueva York.

SOKAL, R. y F. ROHLF, 1979. Biometría. Principios y métodos estadísticos en la investigación biológica. H. Blume Ediciones, Madrid.

STINI, W., 1969. Nutritional stress and growth: Sex difference in adaptive response. American Journal of Physical Anthropology 31 (3): 417-426.

TORRES-ROUFF, C., 2011. Hiding inequality beneath prosperity: Patterns of cranial injury in Middle period San Pedro de Atacama, northern Chile. American Journal of Physical Anthropology 146 (1): 28-37.

TORRES-ROUFF, C. y M. A. COSTA, 2006. Interpersonal violence in prehistoric San Pedro de Atacama, Chile: Behavioral implications of environmental stress. American Journal of Physical Anthropology 130 (1): 60-70.

TORRES-ROUFF, C., M. A. COSTA y A. LLAGOSTERA, 2005. Violence in times of change: The Late Intermediate Period in San Pedro de Atacama. Chungara, Revista de Antropología Chilena $37(1): 75-83$.

TARRAGÓ, M., 1989. Contribución al conocimiento arqueológico de las poblaciones de los oasis de San Pedro de Atacama en relación con los otros pueblos puneños, en especial, el sector septentrional del Valle Calchaquí. Tesis Doctoral, Facultad de Humanidades y Artes, Universidad Nacional de Rosario, Rosario.

VARELA, H., 1997. La población prehistórica de San Pedro de Atacama. Composición, estructura y relaciones biológicas. Tesis Doctoral, Departamento de Ciencias Naturales, Universidad Nacional de Río Cuarto, Río Cuarto.

VARELA, H. y J. COCILOVO, 1996. Los componentes de la variación intramuestral en la población prehistórica de San Pedro de Atacama, Chile. Revista Argentina de Antropología Biológica 1 (1): 219-231.

2000. Structure of the prehistoric population of San Pedro de Atacama. Current Anthropology 41 (1): 125-131.

2002. Genetic drift and gene flow in a prehistoric population of the Azapa Valley and Coast, Chile. American Journal of Physical Anthropology 118 (3): 259-267. 


\section{José Cocilovo, Héctor Varela, María Fuchs}

2009. Microevolución en San Pedro de Atacama (norte de Chile): El cementerio de Quitor. Latin American Antiquity 20 (2): 333-342.

2011. Divergencia fenotípica en los oasis de San Pedro de Atacama, norte de Chile. Estudios Atacameños, Arqueología y Antropología Surandinas 42: 101-112.
VARELA, H., J. COCILOVO y M. A. COSTA, 1990. El dimorfismo sexual en la población prehistórica de San Pedro de Atacama, Chile. Chungara 24-25: 159-166.

WEST, K. y J. MCNAMARA Jr., 1999. Changes in the craniofacial complex from adolescence to midadulthood: A cephalometric study. American Journal of Orthodontics and Dentofacial Orthopedics $115(5): 521-532$. 